\title{
Visual and audiovisual speech perception with color and gray-scale facial images
}

\author{
TIMOTHY R. JORDAN, MAXINE V. MCCOTTER, and SHARON M. THOMAS \\ University of Nottingham, Nottingham, England
}

\begin{abstract}
Research has shown that auditory speech recognition is influenced by the appearance of a talker's face, but the actual nature of this visual information has yet to be established. Here, we report three experiments that investigated visual and audiovisual speech recognition using color, gray-scale, and point-light talking faces (which allowed comparison with the influence of isolated kinematic information). Auditory and visual forms of the syllables /ba/,/bi/,/ga/,/gi/,/va/, and/vi/ were used to produce auditory, visual, congruent, and incongruent audiovisual speech stimuli. Visual speech identification and visual influences on identifying the auditory components of congruent and incongruent audiovisual speech were identical for color and gray-scale faces and were much greater than for point-light faces. These results indicate that luminance, rather than color, underlies visual and audiovisual speech perception and that this information is more than the kinematic information provided by point-light faces. Implications for processing visual and audiovisual speech are discussed.
\end{abstract}

Although speech recognition is often regarded as primarily an auditory process, auditory speech can be influenced strongly by the appearance of a talker's face. For example, seeing the face of a talker can improve intelligibility in a noisy environment (Erber, 1969; MacLeod \& Summerfield, 1987, 1990; Middleweerd \& Plomp, 1987; Sumby \& Pollack, 1954) and help recover a difficult message even when the auditory signal is clear (Reisberg, McLean, \& Goldfield, 1987). The influence of visual speech is demonstrated further by the McGurk effect (McGurk \& MacDonald, 1976) in which different auditory and visual inputs combine to form an illusory percept not present in either modality alone. For example, when auditory /ba/ is presented in synchrony with the lip movements $\mathrm{lga} /$, observers often report a syllable that is a fusion of both sources of information (e.g., "da" or "tha"). Indeed, such is the power of visual information in auditory speech perception that McGurk effects still occur when the auditory signal is clear and unambiguous and when observers are aware of the dubbing process (Liberman, 1982). However, despite these influences, comparatively little is still known about visual speech and the conditions under which it is accurately encoded (see Summerfield, MacLeod, McGrath, \& Brooke, 1989, for a review).

It is generally (and reasonably) assumed that basic visual cues must be encoded for visual speech to be recognized and to affect auditory speech recognition in face-to-

The authorship of this article is alphabetical and reflects the equal contributions to this work by each author. The authors are grateful to Claire Martin and Ken Scott-Brown for useful comments concerning this research and to Duncan Rowland for allowing us to record his face and voice. Correspondence should be addressed to T. R. Jordan, School of Psychology, University of Nottingham, University Park, Nottingham, NG7 2RD, England (e-mail: trj@psychology.nottingham.ac.uk). face interactions (see, e.g., Massaro \& Cohen, 1990; Montgomery \& Jackson, 1983; Petajan, 1984; Summerfield, 1987, 1992; Summerfield \& McGrath, 1984). Most analyses of visual speech address the role of detailed information, such as lip shape (open, rounded, closed, or even protruding), tongue position and shape, and teeth visibility (e.g., Massaro \& Cohen, 1990; Montgomery \& Jackson, 1983; Summerfield et al., 1989; Summerfield \& McGrath, 1984). However, recent findings suggest that pictorial detail is not critical for visual and audiovisual speech recognition. For example, Jordan and Sergeant (1998) investigated effects of image size on visual and audiovisual speech perception using a talking face presented at full size $(100 \%)$ and four smaller sizes $(20 \%$, $10 \%, 5 \%$, and $2.5 \%$ ), all viewed at a constant distance of $1 \mathrm{~m}$. Identification of unimodal visual speech and accuracy of auditory speech report with congruent and incongruent audiovisual speech stimuli was only slightly affected when facial images were reduced to as little as $10 \%$ full size (comparable findings are reported by Jordan \& Sergeant, 2000, using full-size images presented at various distances). As Jordan and Sergeant (1998) point out, this reduction in image size substantially reduces the amount of facial information available to the observer, due to limits in human visual acuity. These findings suggest that detailed pictorial information (e.g., exact shape of the lips, position of the tongue, and visibility of the teeth) is not critical for the perception of visual speech (see also Jordan \& Bevan, 1997; Jordan, Sergeant, Martin, Thomas, \& Thow, 1997).

This idea is further supported by studies using pointlight images where only small points of light are visible and in which no apparent pictorial information is present. This technique has been used in a number of studies in which observers recognized the biological motion pro- 
duced by moving point-light displays as a person performing specific activities (e.g., walking, running, jumping, lifting weights; Bingham, 1987; Johansson, 1973; Kozlowski \& Cutting, 1977) and has also been applied to faces, where expression, age, and gender (Bassili, 1978; Berry, 1990) can be identified. When used for talking faces (Johnson, Rosenblum, \& Saldaña, 1994; Rosenblum, Johnson, \& Saldaña, 1996; Rosenblum \& Saldaña, 1996), a static configuration of reflective dots placed on a blacked-out face could not be identified as a face, suggesting that not enough pictorial facial information was available for static face processing. Nevertheless, when moving, these point-light displays were recognized as unimodal visual speech and exerted powerful influences on congruent and incongruent auditory speech (Johnson et al., 1994; Rosenblum et al., 1996; Rosenblum \& Saldaña, 1996). However, across all these studies, point-light faces did not show the same degree of visual influence as fully illuminated face stimuli. It could be argued that pointlight faces were less influential because the arrangement of points in the displays did not capture all aspects of kinematic visual speech present in a fully illuminated face. Indeed, the optimal point-light configuration for visual and audiovisual speech perception remains an important goal (e.g., Rosenblum et al., 1996; Rosenblum \& Saldaña, 1998). Nevertheless, the evidence suggests that isolated kinematic information is not the only facial cue involved in visual and audiovisual speech perception. Thus, the next line of investigation lies in determining which aspects of a fully illuminated face provide this additional information.

Color is one possibility. For example, color may indicate skin pigmentation and texture, both of which may help to distinguish different parts of the face (e.g., the skin of the lips, tongue, and cheeks), which, in turn, may assist perception of visual speech (e.g., Massaro \& Cohen, 1990; Montgomery \& Jackson, 1983; Summerfield \& McGrath, 1984). Some clues concerning the role of color in general object recognition can be obtained from psychophysical research. For example, a number of studies have reported superior recognition performance with color over gray-scale images (Brodie, Wallace, \& Sharrat, 1991; Davidoff \& Ostergaard, 1988; Humphrey, Goodale, Jakobson, \& Servos, 1994; Ostergaard \& Davidoff, 1985; Price \& Humphreys, 1989; Wurm, Legge, Isenberg, \& Luebker, 1993). Moreover, Price and Humphreys (1989) argue that color is helpful when discriminating among members of a structurally similar class of object. Consequently, color may be highly influential when encoding information from facial images that belong to a class of stimuli composed of different exemplars broadly similar in shape and content. However, the evidence from processing static facial images is mixed. Kemp, Pike, White, and Musselman (1996) found that recognition of unfamiliar faces shown to participants prior to testing was impaired when faces were hue-negated. In contrast, when familiar faces were used, hue-negation did not affect recognition performance or performance in a task requiring detec- tion of feature displacement. More recently, Bruce et al. (2000) found no advantage for unfamiliar face matching using color rather than gray-scale images. However, Lee and Perrett (1997) found that recognition of briefly presented famous faces was more accurate for color images than for their gray-scale counterparts. Moreover, Burt and Perrett (1995) found that color manipulations influenced ratings of the perceived age of faces, and Hill, Bruce, and Akamatsu (1995) showed that rating the sex of faces was influenced by color, although, in this study, presence of color was confounded with three-dimensional (3-D) shape information.

The picture is further complicated by neuropsychological evidence that indicates a separation between static and dynamic face perception. For example, Campbell, Landis, and Regard (1986) reported a double dissociation between face identity/expression judgments and lipreading. Humphreys, Donnelly, and Riddoch (1993) reported 1 patient (H.J.A.) who was prosopagnosic but who could judge expression from a moving point-light face. Campbell (1992) also found that H.J.A. could discriminate speech sounds in moving faces but was unable to identify still pictures of lip speech.

Another reason to suggest that color information may be differentially effective for perception of static and dynamic facial information comes from psychophysical research that suggests that presence of color influences perception of motion. Although some psychophysical studies have shown that color has little effect on perception of motion (e.g., Livingstone \& Hubel, 1987, 1988; Ramachandran \& Gregory, 1978), more recent evidence indicates that chromatic information influences detection of motion under certain viewing conditions (Edwards \& Badcock, 1996; Gegenfurtner \& Hawken, 1996; Krauskopf \& Farell, 1991; Kumar, Beutter, \& Glaser, 1993; Metha, Vingrys, \& Badcock, 1994; Papathomas, Gorea, \& Julesz, 1991; Troscianko, 1994). For example, observers can distinguish motion of briefly presented color patterns independently of luminance variations (Cropper \& Derrington, 1996). Furthermore, Gegenfurtner and Hawken $(1995,1996)$ reported that perception of slow movement (around $4 \mathrm{~Hz}$ ) but not moderate or fast movement $(10$ and $16 \mathrm{~Hz}$ ) is sensitive to chromaticity (see also Hawken, Gegenfurtner, \& Tang, 1994). These values resemble the fundamental frequency observed in talking faces. For example, in a study of the jaw movements made during speech, Ohala (1975) found that the primary spectral peak was around $4 \mathrm{~Hz}$, and Muller and MacLeod (1982) found a similar range for lip-movement frequencies (see also Munhall \& Vatikiotis-Bateson, 1998). Consequently, findings concerning the role of color in basic motion perception raise the possibility that color aids perception of visual speech by assisting perception of facial movement. However, the issue is not resolved. A great deal of research using isoluminant stimuli has suggested that luminance contrast is the dominant cue in motion perception, with color making a minimal contribution (Cavanagh, Tyler, \& Favreau, 1984; Livingstone \& Hubel, 
1987; Ramachandran \& Gregory, 1978). Thus, if color and gray-scale facial images contain essentially the same visible luminance information, no differences in the perception of moving color and gray-scale images would be expected.

In sum, the available evidence concerning the role of color in the perception of static and dynamic faces is equivocal. What is required is a direct contrast between the effects of color and gray-scale faces on the recognition of visual and audiovisual speech. In particular, removing chromaticity from the color face to create a grayscale image (while maintaining identical luminance levels) would allow the relative influences of chromaticity and luminance to be directly assessed. However, this comparison in itself may not fully establish the role of color and gray-scale information. In particular, if grayscale faces are found to have less influence on performance than color faces, it could be argued that color (e.g., skin pigmentation and texture, differences in color between the mouth, nose, and eyes) is a major source of pictorial information for the perception of visual speech. However, residual pictorial information in gray-scale faces (e.g., skin texture, differences in the shading of the teeth, lips, and tongue region) may also be important and provide additional influences over and above those produced by simple facial motion. Comparing performance with color and gray-scale faces and a display type that contains little or no pictorial information but preserves facial motion would provide a benchmark against which to measure any residual visual influence of pictorial information in gray-scale faces, should this exist. An appropriate measure here would be to use point-light images that contain minimal pictorial information and that are derived from points attached to the talker's face, therefore preserving natural facial movement.

Consequently, the present study investigated the role of color in visual and audiovisual speech recognition using three face display types: color, gray-scale, and the point-light images of Rosenblum and Saldaña (1996). If color is important for visual speech perception, visual speech presented in a color talking face should be perceived more accurately and have a greater effect on auditory speech recognition than its gray-scale counterpart. However, if color does not contribute to the perception of visual speech, performance should be similar with color and gray-scale displays. The inclusion of point-light faces allowed the influence of color and gray-scale images to be contrasted with that provided by isolated kinematic information. The most rigorously researched pointlight displays are those used by Rosenblum and Saldaña (1996), and these were used in the present series of experiments. The effects of these three display types on auditory speech perception were examined using congruent audiovisual stimuli in which visual speech matched the auditory signal (as in normal audiovisual speech recognition) and incongruent (McGurk) audiovisual stimuli in which visual speech was dubbed onto an incongruent auditory signal and that offer a stringent test of automatic and perceptual processing (e.g., Jordan \& Bevan, 1997; Jordan \& Sergeant, 1998, 2000; Liberman \& Mattingly, 1985; Rosenblum \& Saldaña, 1992; see also Rosenblum \& Saldaña, 1996). To emphasize the role played by visual speech in these effects and deemphasize effects of redundancy provided by scriptual, expressive, or contextual constraints (e.g., Gailey, 1987; Ronneberg, 1993; Samuelsson \& Ronneberg, 1993; Sudman \& Berger, 1971), stimuli were individual syllables articulated by a face showing a neutral expression.

\section{EXPERIMENT 1}

\section{Method}

Participants. Twenty-four adult native English speakers (age range $18-23$ years) took part in a single $1-\mathrm{h} 45-\mathrm{min}$ session. All participants had normal or corrected-to-normal vision, judged by preexperimental eye chart testing, and good hearing, judged by preexperimental testing with practice auditory stimuli.

Stimuli. The face and voice of a 25 -year-old male was videotaped using a Panasonic S-VHS color video camera. Recordings were made of the talker saying each of the consonant-vowel syllables /ba/,/bi/,/ga/,/gi/,/va/, and/vi/ a number of times. Each syllable was articulated naturally with no artificial emphasis on articulation. The speaker's face was fully illuminated and recorded against a dark background with only the face and neck visible. For point-light stimuli, the same speaker's face was blackened with water-based face paint, the teeth blacked out with theatrical tooth black, and the tongue darkened with food coloring. Following Rosenblum and Saldaña (1996), twenty-eight 3-mm dots constructed from retroreflective tape were attached to the speaker's face, teeth, and tongue using adhesive. Four dots were placed on the upper and lower central incisors, 1 dot on the tongue tip, 6 on the lips, 2 above the lips, 4 on the chin, 8 on the cheeks, 2 on the jaw, and 1 on the nose tip (see Rosenblum \& Saldaña, 1996).

For both full-face and point-light recordings, the talker sat $1.5 \mathrm{~m}$ from the camera. One example of each syllable was selected from the full-face and point-light recordings and captured onto computer. The digitized clips were edited to produce the following stimuli:

1. Auditory speech: auditory /ba/,/bi/,/ga/,/gi/,/va/, and /vi/, all presented with a static face of the talker with mouth closed. ${ }^{\prime}$

2. Visual speech: visual /ba/,/bi/,/ga/,/gi/,/va/, and /vi/, all with no auditory signal.

3. Congruent audiovisual speech: audiovisual /ba/, /bi/, /ga/, /gi/,/va/, and /vi/.

4. Incongruent audiovisual speech: auditory /ba/paired with visual /ga/, auditory / bi/paired with visual /gi/, auditory/ga/paired with visual /ba/, auditory /gi/ paired with visual /bi/, auditory /ba/ paired with visual /va/, auditory /bi/ paired with visual/vi/. These combinations are known to produce powerful McGurk effects (e.g., Green, Kuhl, Meltzoff, \& Stevens, 1991; Jordan \& Bevan, 1997).

Point-light images were adjusted for brightness and contrast and filtered to ensure that only the reflective dots (i.e., none of the surface of the face) were visible. The sound intensity of all auditory, congruent, and incongruent stimuli was equalized, and any background noise prior to speech was removed. The clips were recorded back onto S-VHS tape, one tape for the full face stimuli and one tape for the point-light stimuli. Tapes were played back on a highresolution visual display screen with auditory signals presented via two adjacent Spendor S100 studio broadcast loudspeakers at a sound level of approximately $55 \mathrm{~dB}$. The monitor saturation settings were adjusted to produce naturalistic levels of facial color for color images, as judged by observers who matched screen color with the facial color of the individual filmed. Saturation settings were readjusted to produce gray-scale images in which all color in- 
formation had been removed. Color and gray-scale images were matched for luminance using a Cambridge Research Systems OptiCAL Gamma correction photometer. Point-light stimuli were presented at the same monitor settings as gray-scale images. For visual, congruent, and incongruent stimuli, the face remained static for $2 \mathrm{sec}$ before onset of articulation. For auditory speech stimuli, the face remained static throughout (with mouth closed); onset of the auditory signal was $2 \mathrm{sec}$ after visual stimulus onset. Each trial in the experiment lasted $4 \mathrm{sec}$, followed by a 5 -sec blank.

Design. Each tape comprised eight cycles of the 24 stimuli, with a different random order for each cycle. Each tape started with a display of all 24 stimuli, shown as practice items. Each participant saw all three display conditions (color, gray-scale, point light), each separated by a break of approximately $5 \mathrm{~min}$. The order in which these conditions were presented was counterbalanced across participants.

Procedure. Each participant was seated at a table approximately $1.5 \mathrm{~m}$ in front of the display screen, with his/her head level with the screen and supported on a chinrest. Each participant had a booklet placed on the table in front of him/her containing 12 response alternatives ("ba," "bi," "bga," "bgi," "da," "di," "ga," "gi," "tha," "thi," "va," "vi") for each trial. Pretesting had established that these 12 responses constituted more than $97 \%$ of participants' perceptions of the stimuli used in this experiment. The participants were instructed to look at the screen and listen throughout the trials and to make their responses by marking the syllable they heard (with the emphasis in the instructions being on heard) on their response sheet. The participants were instructed to report the syllable they thought was being articulated when presented with visual speech trials.

\section{Results}

Mean identification accuracy for visual, congruent, and incongruent speech is shown in Figure 1. The patterns of performance suggest similar effects with color and grayscale images but different effects with point-light images. A preliminary analysis of variance (ANOVA) including the factor of presentation order of display type (color, gray-scale, point light) revealed no main effect or interaction involving this factor (all $p \mathrm{~s}>.40$ ). Thus, all subsequent analyses proceeded without presentation order as a factor.

Auditory stimuli were identified with $84 \%$ accuracy across all display types $[F(2,44)=0.87, p>.50]$. Thus, auditory stimuli were essentially equally intelligible in each display type. ${ }^{2}$ One-way ANOVAs with the factor of display type were conducted for visual, congruent, and incongruent speech conditions. A main effect of display type was found for all three conditions [visual, $F(2,46)=$ $119.88, p<.0001$; congruent, $F(2,46)=15.60, p<.0001$; incongruent, $F(2,46)=59.23, p<.0001]$. Newman-Keuls tests for visual and congruent conditions showed no difference between accuracy with color and gray-scale displays ( $p s>.30)$, but both produced higher accuracy than point-light displays ( $p$ s $<.0001$ ). Newman-Keuls tests for incongruent conditions showed no difference in accuracy for color and gray-scale displays $(p>.80)$, but both produced lower accuracy than point-light displays (i.e., more McGurk responses were made; $p s<.0001$ ).

The data obtained with congruent and incongruent stimuli were compared with those obtained with the same auditory signals in the auditory speech condition. For congruent stimuli, an ANOVA with factors of presentation condition (congruent, auditory) and display type revealed a main effect of display type $[F(2,46)=9.38, p<.0001]$, which interacted with speech condition $[F(2,46)=9.27$, $p<.0005]$. Performance was more accurate in the congruent condition when color and gray-scale displays were used (Newman-Keuls, both $p$ s $<.002$ ). However, no difference was found between congruent and auditory speech

\section{$\square$ Auditory $\square$ Visual $\mathbf{m}$ Congruent $\square$ Incongruent}

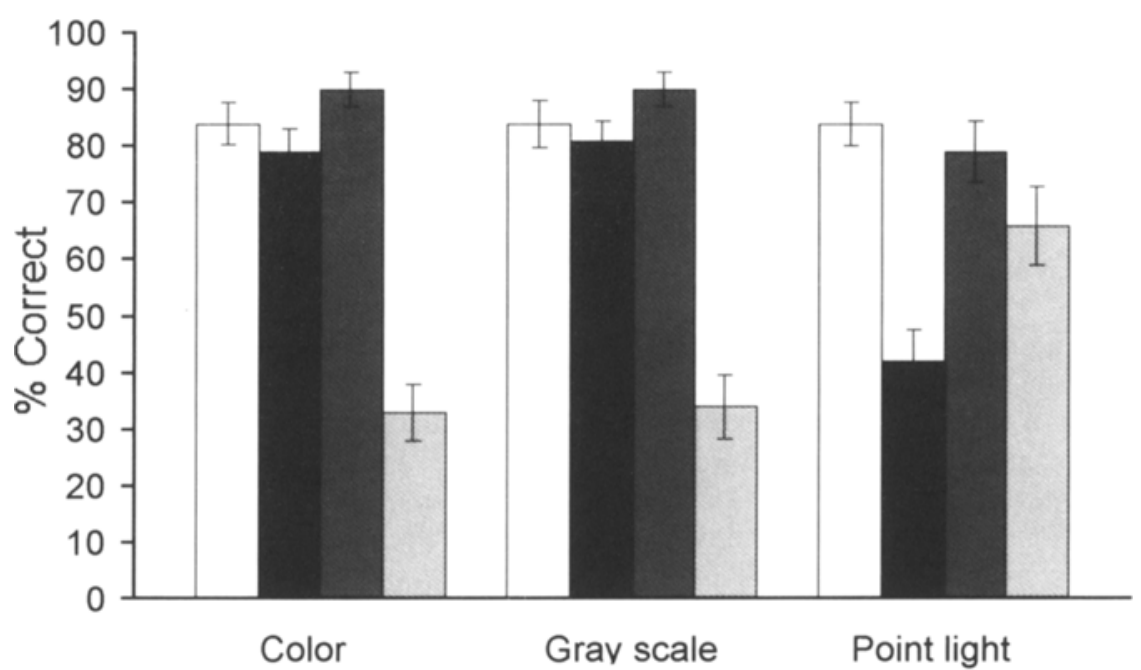

\section{Display Type}

Figure 1. Mean percentage of auditory and visual stimuli and the auditory component of congruent and incongruent audiovisual stimuli correctly identified in Experiment 1 for each display type. 
in the point-light condition $(p>.05)$. For incongruent stimuli, a similar ANOVA revealed main effects of presentation condition $[F(1,23)=93.30, p<.0001]$ and display type $[F(2,46)=49.61, p<.0001]$ and an interaction between these two factors $[F(2,46)=29.35, p<.0001]$. Newman-Keuls comparisons showed fewer correct responses in all display types for incongruent audiovisual stimuli when compared with the auditory speech condition $(p s<.0001)$, although this deficit was smaller for point-light displays.

\section{Discussion}

The major finding of Experiment 1 was that, while perceptibility of visual speech and its influence on auditory speech perception was evident for point-light images, these effects were greater for color and gray-scale faces, which produced essentially identical patterns of performance. In particular, visual speech was identified equally accurately when presented in color and gray-scale faces and more accurately than in point-light images. Moreover, color and gray-scale faces produced the same levels of accuracy for congruent audiovisual stimuli, and both provided the same amount of improvement in performance when compared with the auditory speech condition; congruent audiovisual speech presented in point-light images produced lower report accuracy and no improvement in performance when compared with the auditory speech condition (see also Rosenblum \& Saldaña, 1996). Furthermore, color and gray-scale faces produced the same levels of accuracy for incongruent audiovisual stimuli and the same amount of impairment to performance when compared with the auditory condition; incongruent audiovisual speech presented in point-light images produced higher report accuracy and less impairment in performance when compared with the auditory speech condition (see also Rosenblum \& Saldaña, 1996). These findings suggest that, while visual and audiovisual speech is encoded effectively when observed in full-color talking faces, color information plays no role in this process. However, this issue was investigated further in Experiments 2 and 3 .

\section{EXPERIMENTS 2 AND 3}

A great deal of research suggests that the influence of visual speech on auditory speech recognition increases when the signal-to-noise ratio of the auditory signal decreases (e.g., Jordan \& Sergeant, 1998; MacLeod \& Summerfield, 1987, 1990; Middleweerd \& Plomp, 1987; Sumby \& Pollack, 1954). For example, Jordan and Sergeant (1998) found that, although manipulating the quality of visual speech (in this case, by changing the size of facial images) produced changes in the influence of visual speech on congruent and incongruent auditory speech recognition in a noise-free environment, these effects were more widespread and generally more substantial when auditory signals were presented in a background of white noise. In a similar vein, presenting auditory signals in white noise may provide a more sensitive test of changes in visual speech influence across the color, gray-scale, and point-light images used in Experiment 1.

Thus, in Experiments 2 and 3, auditory signals were presented in a background of white noise. Moreover, in order to provide appropriate "headroom" for revealing individual effects of display type (should they exist) on the influences exerted by congruent and incongruent visual speech, different noise levels were used in each experiment. In Experiment 2, the primary concern was the effect of display type on performance with congruent audiovisual stimuli. Consequently, the noise level used was aimed at producing $40 \%$ correct report in the auditory condition, which allowed improvements in the congruent audiovisual condition to vary between display types within a range of $60 \%$. In Experiment 3, the primary concern was the effect of display type on performance with incongruent audiovisual stimuli. Consequently, the noise level used was aimed at producing $68.33 \%$ correct report in the auditory condition, which allowed impairments in the incongruent audiovisual condition to vary between display types within a range of $60 \%(8.33 \%$ is the minimum performance level possible with 12 alternative responses). Although these were the primary concerns for Experiments 2 and 3, congruent and incongruent stimuli were shown in each experiment for completeness.

\section{Method}

Participants. Twenty-four new participants from the same population as Experiment 1 took part in each of Experiments 2 and 3. No participant took part in both experiments, and none had taken part in Experiment 1.

Stimuli. The stimuli used in Experiment 1 were used in Experiments 2 and 3 . However, these stimuli were now presented in a background of continuous white noise at a sound level of $70 \mathrm{~dB}$ in Experiment 2 and $55 \mathrm{~dB}$ in Experiment 3 . All remaining aspects of Experiments 2 and 3 were the same as in Experiment 1.

\section{Results}

Experiment 2. Mean identification accuracy for visual, congruent, and incongruent speech is shown in Figure 2 . The patterns of performance suggest similar effects when color and gray-scale images are used as stimuli but different effects when point-light images are used. A preliminary ANOVA including presentation order of display type (color, gray-scale, point light) revealed no main effect or interaction involving this factor (all $p s>.80$ ). Thus, all subsequent analyses proceeded without this factor.

Auditory stimuli were identified with $39 \%$ accuracy across all display types $[F(2,46)=0.20, p>.40]$, which closely approximated our goal of $40 \%$. One-way ANOVAs with the factor of display type were conducted for visual, congruent, and incongruent speech conditions. A main effect of display type was found for all three conditions [visual, $F(2,46)=109.31, p<.0001$; congruent, $F(2,46)=$ $29.50, p<.0001$; incongruent, $F(2,46)=4.69, p<.015]$. Newman-Keuls tests for visual and congruent conditions showed no difference between accuracy with color and gray-scale displays ( $p s>.30)$, but both produced higher 


\section{$\square$ Auditory $\mathbf{a}$ Visual $\mathbf{m}$ Congruent $\square$ Incongruent}

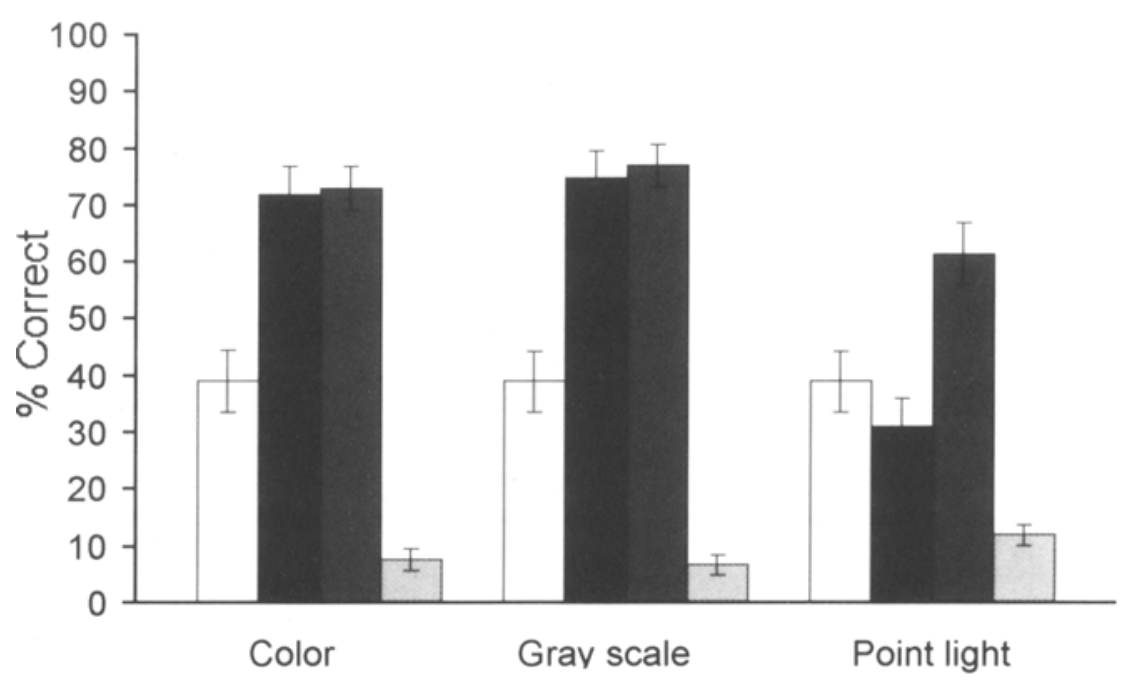

\section{Display Type}

Figure 2. Mean percentage of auditory and visual stimuli and the auditory component of congruent and incongruent audiovisual stimuli correctly identified in Experiment 2 for each display type.

accuracy than point-light displays $(p s<.0001)$. NewmanKeuls tests for incongruent conditions showed no difference in accuracy for color and gray-scale displays $(p>.50)$, but both produced lower accuracy than point-light displays (i.e., more McGurk responses were made; $p s<.01$ ).

The data obtained with congruent and incongruent stimuli were compared with those obtained with the same auditory signals in the auditory speech condition. For congruent stimuli, an ANOVA with factors of presentation condition (congruent, auditory) and display type revealed main effects of presentation condition $[F(1,23)=$ $366.84, p<.0001]$ and display type $[F(2,46)=16.07$, $p<.0001]$ and an interaction between these two factors $[F(2,46)=11.12, p<.0001]$. Newman-Keuls tests showed that congruent visual speech improved accuracy for all display types (all $p s<.0001$ ), although the improvement was less with point-light displays. For incongruent stimuli, a similar ANOVA revealed a main effect of presentation condition $[F(1,23)=105.71, p<.0001]$, which interacted with display type $[F(2,46)=10.22, p<.0001]$. Newman-Keuls tests showed that incongruent visual speech impaired accuracy for all display types (all $p \mathrm{~s}<$ .0001 ), although the impairment was less with pointlight displays.

Experiment 3. Mean identification accuracy for visual, congruent, and incongruent speech tokens is shown in Figure 3. The patterns of performance suggest similar effects when color and gray-scale images are used as stimuli but different effects when point-light images are used. A preliminary ANOVA including presentation order revealed no main effect or interaction involving this factor (all $p s>.40$ ). Thus, all subsequent analyses proceeded without this factor.
Auditory stimuli were identified with $67 \%$ accuracy across all display types $[F(2,46)=2.23, p>.11]$, which closely approximated our goal of $68.33 \%$. One-way ANOVAs with the factor of display type were conducted for visual, congruent, and incongruent speech conditions. A main effect of display type was found for all three conditions [visual, $F(2,46)=189.05, p<.0001$; congruent, $F(2,46)=39.11, p<.0001$; incongruent, $F(2,46)=8.17$, $p<.0009]$. Newman-Keuls tests for visual and congruent conditions showed no difference in accuracy for color and gray-scale displays $(p s>.50)$, but both produced higher accuracy than point-light displays $(p s<.0001)$. NewmanKeuls tests for incongruent conditions showed no difference in accuracy for color and gray-scale displays $(p>.90)$, but both produced lower accuracy than point-light displays (i.e., more McGurk responses were made; $p s<.005$ ).

The data obtained with congruent and incongruent stimuli were compared with those obtained with the same auditory signals in the auditory speech condition. For congruent stimuli, an ANOVA with factors of presentation condition (congruent, auditory) and display type revealed main effects of presentation condition $[F(1,23)=$ $77.87, p<.0001]$ and display type $[F(2,46)=17.83, p<$ $.0001]$ and an interaction between these factors $[F(2,46)=$ $21.67, p<.0001]$. Newman-Keuls tests showed that congruent visual speech improved accuracy for color and gray-scale displays (both $p$ s $<.0001$ ) but not point-light displays $(p>.17)$. For incongruent stimuli, a similar ANOVA revealed main effects of presentation condition $[F(1,23)=104.12, p<.0001]$ and display type $[F(2,46)=$ $4.7, p<.01]$ and an interaction between these factors $[F(2,46)=9.02, p<.0001]$. Newman-Keuls tests showed that incongruent visual speech impaired accuracy for all 


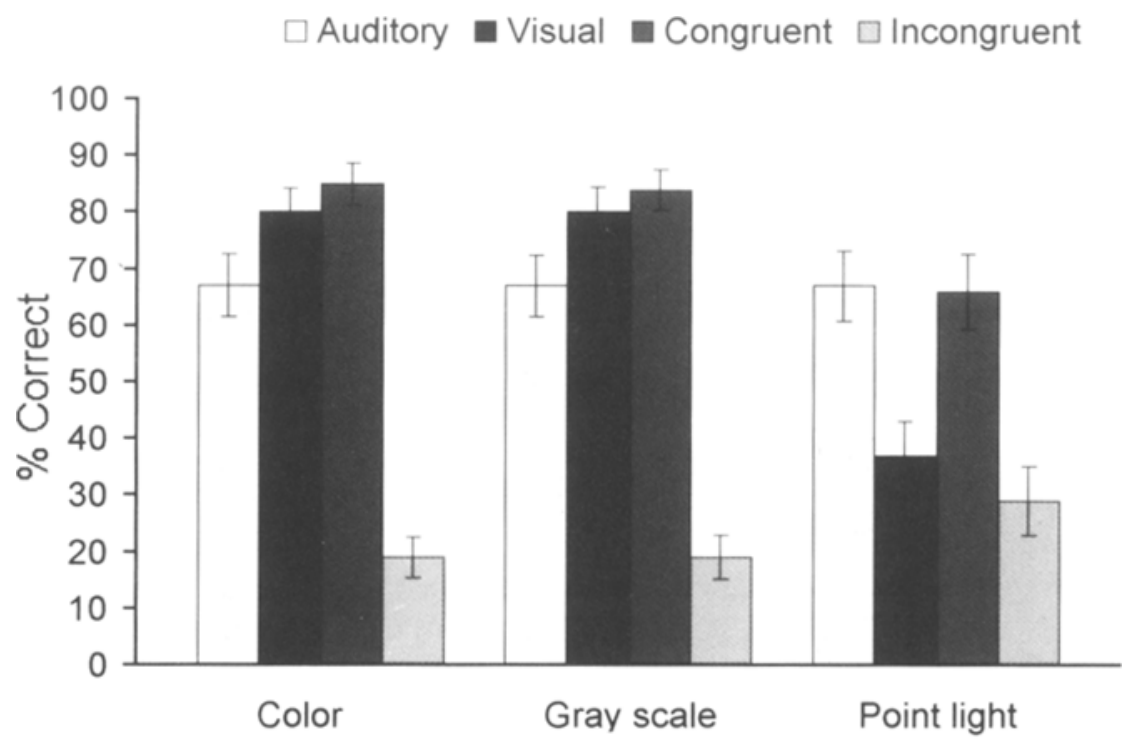

\title{
Display Type
}

\begin{abstract}
Figure 3. Mean percentage of auditory and visual stimuli and the auditory component of congruent and incongruent audiovisual stimuli correctly identified in Experiment 3 for each display type.
\end{abstract}

display types (all $p \mathrm{~s}<.0001$ ), although the impairment was less for point-light displays.

\section{Discussion}

The results of Experiments 2 and 3 consolidate the finding that, while perceptibility of visual speech and its influence on auditory speech perception was evident for point-light images, these effects were more evident for color and gray-scale faces, which produced essentially identical patterns of performance. Thus, while the visual speech present in naturalistic, full-color talking faces is more perceptible and influential than the isolated kinematic information present in point-light images, the perception of visual and audiovisual speech in naturalistic, full-color talking faces may not actually involve perception of color.

Before discussing these findings further, the results obtained with our benchmark condition (point-light displays) are worthy of specific attention. Our findings for point-light displays are similar to those of Rosenblum and Saldaña (1996), who demonstrated that, although isolated kinematic information exerted a visual influence on perception of auditory speech, this was less than for fully illuminated (color) faces. It could be argued that the pointlight configuration used by Rosenblum and colleagues (Johnson et al., 1994; Rosenblum et al., 1996; Rosenblum \& Saldaña, 1996, 1998) and in the present study does not capture all the salient aspects of kinematic visual speech information in fully illuminated faces (color or gray-scale). For example, moving facial features (e.g., jaw, lips) and moving facial tissue (e.g., wrinkling of the skin on lips and cheeks) are likely to provide many additional points of motion information for visual speech perception that are not represented generally in point-light displays (see General Discussion section). Perhaps if the sheer number of points of motion in point-light displays were increased and their optimal arrangement determined (see Rosenblum et al., 1996) to match the kinematic information provided by normal talking faces, visual speech in point-light faces would be more perceptible and influential. However, when considering the locations of these additional points of motion, note that the findings reported in this article suggest that the salient aspects of kinematic visual speech information in fully illuminated faces are not determined by the presence of color.

\section{GENERAL DISCUSSION}

The major point to emerge from the findings of this study is that, despite obvious differences in color that exist across the various components of talking faces (particularly visible articulators such as teeth, lips, tongue, and jaw), information derived from color does not contribute to the recognition of visual and audiovisual speech. Moreover, the naturalistic levels of facial color used make it highly unlikely that the similar findings observed with color and gray-scale faces reflect the influence of weakly effective color images. Indeed, the effects obtained with color images closely resemble (and sometimes exceed) those obtained in other studies using color displays (e.g., Binnie \& Barrager, 1969; Binnie, Montgomery, \& Jackson, 1974; Brannon, 1961; Green \& Gerdeman, 1995; Green et al., 1991; Hutton, 1959; IJsseldijk, 1992; Walden, Prosek, Montgomery, Scherr, \& Jones, 1977; Walden, 
Prosek, \& Worthington, 1974). Thus, when attempting to determine which aspects of talking faces contribute to visual speech, it would seem wise to focus on aspects present in gray-scale facial images. But which aspects of gray-scale facial information may underlie the effects we observed?

\section{Luminance Variations and Their Spatial Distribution Across the Face}

Variations in basic luminance are present in color and gray-scale facial images and provide many possible sources of information for visual speech perception. For example, teeth appear lighter than the rest of the mouth region, and the inside of the mouth appears darker than the lips. In addition, the tongue is lighter than the lips, which, in turn, appear darker than surrounding facial tissue. Luminance also helps specify shadow boundaries that may enhance perception of the contours of these facial features (e.g., Cavanagh \& Leclerc, 1989) and help specify the parameters of extraoral features, such as eyes, jaw, chin, and cheeks, which may also contribute to recognition of visual and audiovisual speech (Greenberg \& Bode, 1968; Guiard-Marigny, Ostry, \& Benoit, 1995; Larr, 1959; Smeele, Hahnlen, Stevens, Kuhl, \& Meltzoff, 1995; Stone, 1957; Vatikiotis-Bateson, Eigsti, Yano, \& Munhall, 1998).

Differences in luminance also indicate patterns of shading that can help specify 3-D shape cues (e.g., Ramachandran, 1988). Indeed, research has suggested that perception of 3-D shape from shading depends primarily on luminance contrast, with color providing no additional input (Cavanagh, 1987; Cavanagh \& Leclerc, 1989; Livingstone \& Hubel, 1987; Ramachandran, 1988). Moreover, evidence from face recognition research suggests that recovery of 3-D shape from shading has a role in sex (Bruce \& Langton, 1994) and identity (Hill \& Bruce, 1996) judgments for static faces. In a similar vein, perception of visual and audiovisual speech may also rely on shading (provided by luminance differences) in the recovery of 3-D shape information. Indeed, shading information present in the moving jaw, chin, lips, teeth, and tongue may provide important depth cues in talking faces. For example, in contrast to the darker regions of the tongue inside the mouth, the lighter areas on the tip of the tongue as it protrudes may provide cues to the position of the tongue in the depth plane, which may be important for perception of visual speech (e.g., Massaro \& Cohen, 1990). Depth perception may also be important for identifying relative motion-for example, between the lips and teeth (Rosenblum \& Saldaña, 1998). Recovery of 3-D shape from shading also raises the possibility that 2-D patterns of shading in talking faces may facilitate access to stored gray-scale 3-D representations of dynamic visual speech. Jordan et al. (1997) have provided evidence suggesting that access to representations for visual speech is viewpoint invariant across full-face, threequarter, and profile views. If this were the case, shading (provided by luminance differences) may contribute to the formation of stored 3-D representations of visual speech and provide information about the facial characteristics and dynamic facial information involved in visual and audiovisual speech recognition from a range of different viewpoints. Indeed, access to 3-D information through shading could provide basic visual information for the perception of visual and audiovisual speech across a wide variety of viewing conditions. For example, when objects are presented in unconventional views (e.g., when the object's principal axis of symmetry is foreshortened), shading may play an important role in the recovery of depth information (Marr \& Nishihara, 1978). Similarly, pictorial information is likely to be lost when talking faces are viewed at a distance (Jordan \& Sergeant, 2000; see also Jordan \& Sergeant, 1998) or in profile (Jordan et al., 1997), but basic areas of shading (e.g., the jaw, cheeks, and mouth) may remain visible, which could facilitate access to 3-D representations of visual speech.

However, perception of visual and audiovisual speech may be sensitive not just to simple luminance differences (e.g., dark vs. light) but also to the spatial distribution of these differences across the face. This notion is supported by recent evidence (Campbell, 1996; Campbell, de Haan, \& Brooks, 1997) showing that reversing the brightness variations of a talking face by presenting the image in photonegative severely disrupts performance with visual speech and fails to generate McGurk responses. In a more recent investigation (Kanzaki \& Campbell, 1999), using a different speaker, the effects of brightness reversal were less disruptive, although still evident. Thus, visual and audiovisual speech recognition may be sensitive to not only the basic contours of the face and the layout of the facial features (which are preserved in negative images) but also the characteristic patterns of luminance across the face. One possible implication of this finding for understanding visual and audiovisual speech recognition is that negation of talking facial images disrupts the recovery of 3-D shape from patterns of shading (e.g., Kemp et al., 1996), which in turn could impair access to stored representations of visual speech. Furthermore, if (as previously argued) luminance variations are an intrinsic aspect of stored representations of visual speech, these representations may encompass both basic luminance differences and more complex variations specified by the relative areas of dark and light in talking faces. We are currently investigating this issue.

\section{Potential Benefits of Visual Speech Perception Operating Via Gray-Scale Information}

A system of visual speech perception that encodes gray-scale information rather than color information may be better suited to accommodate the range of lighting conditions under which visual speech is typically encoded, including bright sunlight, artificial light, and near darkness. Specifically, luminance differences can be perceived under conditions of photopic (low-illumination) and scotopic (high-illumination) vision. Indeed, the perceptual phenomenon of lightness constancy demon- 
strates that perceived lightness of objects remains constant even under several different levels of illumination (e.g., Jacobsen \& Gilchrist, 1988). However, color differences become difficult to detect at lower levels of illumination where cone receptors in the retina (which are involved in color perception) fail to function (see, e.g., Cornsweet, 1970; Sekuler \& Blake, 1994). In addition, changes in perceived color also occur when the quality, rather than quantity, of illumination has changed (e.g., Helson, Judd, \& Wilson, 1956; Hurvich, 1981). Thus, in order to process visual speech effectively across different viewing conditions, visual and audiovisual speech perception may have evolved to make use of luminance cues because of their relative robustness across different levels of illumination.

\section{Comparison With Static Face Recognition}

In contrast to our findings for visual and audiovisual speech recognition, color has been shown to influence judgments of the identity, age, and gender of static faces (Burt \& Perrett, 1995; Hill et al., 1995; Kemp et al., 1996; Lee \& Perrett, 1997), although the role of color in processing static faces remains to be fully resolved (see introduction). This adds support to the notion that the information used in static face tasks differs from that used in the recognition of visual and audiovisual speech and may be explained by differences in the nature of each task (see Jordan \& Bevan, 1997, and Jordan \& Thomas, 1999, for further discussion of this point). In particular, judgments of age, gender, and identity may be derived from a system of face processing, which, under normal everyday conditions, requires sufficient information to determine an appropriate judgment for each of an innumerable range of structurally similar inputs. Thus, all available facial information, including color and other aspects of surface detail (see Bruce et al., 1991; Bruce \& Langton, 1994; Price \& Humphreys, 1989), may be useful in this type of task. In contrast, accurate visual speech recognition requires only sufficient information to distinguish between relatively few visual speech possibilities (e.g., spoken English contains 40 or so phonemes, which, from Walden et al., 1977, are represented by even fewer visemes). Thus, although the present findings cannot fully clarify the issue of dissociation of function between processing of static and dynamic faces, they do underscore the notion that dynamic visual speech recognition and static face processing may utilize different sources of facial information.

\section{REFERENCES}

BassiLi, J. N. (1978). Facial motion in the perception of faces and of emotional expression. Journal of Experimental Psychology: Human Perception \& Performance, 4, 373-379.

BERRY, D. S. (1990). What can a moving face tell us? Journal of Personality \& Social Psychology, 58, 1004-1014.

Bingham, G. P. (1987). Scaling and kinematic form: Further investigations on the visual perception of lifted weight. Journal of Experimental Psychology: Human Perception \& Performance, 13, 155-177.

BinNie, C. A., \& Barrager, D. C. (1969, November). Bi-sensoryestablished articulation functions for normal hearing and sensori- neural hearing loss patients. Paper presented at the annual convention of the American Speech and Hearing Association, Chicago.

Binnie, C. A., Montgomery, A. A., \& Jackson, P. L. (1974). Auditory and visual contributions to the perception of consonants. Journal of Speech \& Hearing Research, 17, 619-630.

BRANNON, J. B. (1961). Speechreading of various speech materials. Journal of Speech \& Hearing Disorders, 26, 348-353.

Brodie, E. E., Wallace, A. M., \& Sharrat, B. (1991). Effect of surface characteristics and style of production on naming and verification of pictorial stimuli. American Journal of Psychology, 104, 517-545.

Bruce, V., Healey, P., Burton, M., Doyle, T., Coombes, A., \& LiNNEY, A. (1991). Recognising facial surfaces. Perception, 20, 755-769.

Bruce, V., Henderson, Z., Greenwood, K., Hancock, P., Burton, M., \& MilleR, P. (2000). Verification of face identities from images captured on video. Journal of Experimental Psychology: Applied, 5, 339360.

Bruce, V., \& Langton, S. (1994). The use of pigmentation and shading information in recognising the sex and identities of faces. Perception, 23, 803-822.

Burt, D. M., \& PerretT, D. I. (1995). Perception of age in adult caucasian male faces: Computer graphic manipulation of shape and colour information. Proceedings of the Royal Society of London: Series $B, 259,137-143$.

CAMPBELL, R. (1992). The neuropsychology of lipreading. Philosophical Transactions of the Royal Society of London: Series B, 335, 39-45.

CampBell, R. (1996). Seeing speech in space and time: Psychological and neurological findings. In Proceedings of the Fourth International Conference on Spoken Language Processing (pp. 1493-1496). Philadelphia: ICSLP.

CAMpBELl, R., DE HAAN, E., \& Brooks, B. (1997). Space and time in the identification of spoken syllables (Department of Human Communication Science, Work in Progress, No. 7). London: University College.

Campbell, R., Landis, T., \& Regard, M. (1986). Face recognition and lipreading: A neurological dissociation. Brain, 109, 509-521.

CAVANAGH, P. (1987). Reconstructing the third dimension: Interactions between colour, texture, motion, binocular disparity and shape. Computer Vision, Graphics \& Image Processing, 37, 171-195.

CAVANAGH, P., \& LECLERC, Y. G. (1989). Shape from shadows. Journal of Experimental Psychology: Human Perception \& Performance, 15, 3-27.

Cavanagh, P., Tyler, C. W., \& Favreau, O. E. (1984). Perceived velocity of moving chromatic gratings. Journal of the Optical Society of America A, 1, 893-899.

Connsweet, T. N. (1970). Visual perception. New York: Academic Press.

Cropper, S. J., \& Derrington, A. M. (1996). Rapid colour specific detection of motion in human vision. Nature, 379, $72-74$

Davidoff, J. B., \& OstergaARd, A. L. (1988). The role of colour in categorical judgments. Quarterly Journal of Experimental Psychology, 40A, 533-544.

EDWARDS, M., \& BADCOCK, D. (1996). Global motion perception: Interaction of chromatic and luminance signals. Vision Research, 36, 2423-2431

ERBER, N. (1969). Interaction of audition and vision in the recognition of oral speech stimuli. Journal of Speech \& Hearing Research, 12, 423-425.

GaILEY, L. (1987). Psychological parameters of lip reading skill. In B. Dodd \& R. Campbell (Eds.), Hearing by eye: The psychology of lip reading (pp. 115-141). London: Erlbaum.

GegenfuRtner, K. R., \& HAWken, M. J. (1995). Temporal and chromatic properties of motion mechanisms. Vision Research, 35, 1547-1563,

GegenfurtNER, K. R., \& HAWKEN, M. J. (1996). Interaction of motion and color in the visual pathways. Trends in Neurosciences, 19, 394-401.

Green, K. P., \& Gerdeman, A. (1995). Cross-modal discrepancies in coarticulation and the integration of speech information: The McGurk effect with mismatched vowels. Journal of Experimental Psychology: Human Perception \& Performance, 6, 1409-1426.

Green, K. P., Kuhl, P. K., Meltzoff, A. N., \& Stevens, E. B. (1991). Integrating speech information across talkers, gender, and sensory modality: Female faces and male voices in the McGurk effect. Perception \& Psychophysics, 50, 524-536.

GreEnBeRG, H. J., \& BoDE, D. L. (1968). Visual discrimination of consonants. Journal of Speech \& Hearing Research, 11, 869-874.

Guiard-Marigny, T., Ostry, D. J., \& Benoit, C. (1995). Speech in- 
telligibility of synthetic lips and jaw. Proceedings of the 13th International Congress of Phonetic Sciences, 3, 222-225.

Hawken, M. J., Gegenfurtner, K. R., \& TANG, C. (1994). Contrast dependence of colour and luminance motion mechanisms in human vision. Nature, 367, 268-270.

Helson, H., JudD, D. B., \& Wilson, M. (1956). Color rendition with fluorescent sources of illumination. Illuminating Engineering, 51, 329-346.

HiLl, H., \& BruCE, V. (1996). Effects of lighting on the perception of facial surfaces. Journal of Experimental Psychology: Human Perception \& Performance, 22, 986-1004.

Hill, H., Bruce, V., \& Akamatsu, S. (1995). Perceiving the sex and race of faces: The role of shape and colour. Proceedings of the Royal Society of London: Series B, 261, 367-373.

Humphrey, G. K., Goodale, M. A., Jakobson, L. S., \& Servos, P. (1994). The role of surface information in object recognition: Studies of a visual form agnosic and normal subjects. Perception, 23, 1457-1481.

Humphreys, G. W., DonNelly, N., \& RidDOCh, J. (1993). Expression is computed separately from facial identity and it is computed separately for moving and static faces: Neuropsychological evidence. Neuropsychologia, 21, 173-181.

Hurvich, L. M. (1981). Color vision. Sunderland, MA.: Sinauer.

Hutton, C. (1959). Combining auditory and visual stimuli in aural rehabilitation. Volta Review, 61, 316-319.

IJsSELDIJK, F. J. (1992). Speechreading performance under different conditions of video image, repetition, and speech rate. Journal of Speech \& Hearing Research, 35, 466-471.

JACOBSEN, A., \& GILCHRIST, A. (1988). The ratio principle holds over a million-to-one range of illumination. Perception \& Psychophysics, 43, $1-6$.

JoHANsSON, G. (1973). Visual perception of biological motion and a model for its analysis. Perception \& Psychophysics, 14, 201-211.

Johnson, J. A., Rosenblum, L. D., \& Saldaña, H. M. (1994). The contribution of a reduced visual image to speech perception in noise [Abstract]. Journal of the Acoustical Society of America, 95 (5, Pt. 2), 3009.

JORDAN, T. R., \& BEVAN, K. (1997). Seeing and hearing rotated faces: Influences of facial orientation on visual and audiovisual speech recognition. Journal of Experimental Psychology: Human Perception \& Performance, 23, 388-403.

JoRDAN, T. R., \& SERGEANT, P. C. (1998). Effects of facial image size on visual and audio visual speech recognition. In R. Campbell, B. Dodd, \& D. Burnham (Eds.), Hearing by eye: Part 2. The psychology of speechreading and audiovisual speech (pp. 155-176). London: Taylor \& Francis.

Jordan, T. R., \& SERGEANT, P. C. (2000). Effects of distance on visual and audiovisual speech recognition. Language \& Speech, 43, 107-124.

Jordan, T. R., Sergeant, P. C., Martin, C., Thomas, S. M., \& THOw, E. (1997). Effects of horizontal viewing angle on visual and audiovisual speech perception. In Proceedings of IEEE International Conference on Computational Cybernetics and Simulation (pp. 16261631). Washington, DC: IEEE.

Jordan, T. R., \& Thomas, S. M. (1999). Effects of horizontal viewing angle on visual and audiovisual speech recognition. Manuscript submitted for publication.

KANZAKI, R., \& CAMPBELL, R. (1999, August). Effects of facial brightness reversal on visual and audiovisual speech perception. Paper presented at the Audio Visual Speech Processing Conference, University of California, Santa Cruz.

Kemp, R., Pike, G., White, P., \& Musselman, A. (1996). Perception and recognition of normal and negative faces: The role of shape from shading and pigmentation cues. Perception, 25, 37-52.

Kozlowski, L. T., \& CutTING, J. E. (1977). Recognizing the sex of a walker from a dynamic point-light display. Perception \& Psychophysics, 21, 575-580.

KraUSKOPF, J., \& FARELL, B. (1991). Vernier acuity: Effects of chromatic content, blur, and contrast. Vision Research, 31, 735-749.

Kumar, T., Beutter, B. R., \& Glaser, D. A. (1993). Perceived motion of a colored spot in a noisy chromatic background. Perception, 22, 1205-1226.
LARR, A. L. (1959). Speechreading through closed-circuit television. Volta Review, 61, 19-21.

Lee, K. J., \& Perrett, D. (1997). Presentation time measures of the effects of manipulations in color space on discrimination of famous faces. Perception, 26, 733-752.

Liberman, A. M. (1982). On finding that speech is special. American Psychologist, 37, 148-167.

Liberman, A. M., \& Mattingly, I. G. (1985). The motor theory of speech perception revised. Cognition, 21, 1-36.

Livingstone, M. S., \& Hubel, D. H. (1987). Psychophysical evidence for separate channels for the perception of form, color, movement and depth. Journal of Neuroscience, 7, 3416-3468.

Livingstone, M. S., \& HubEL, D. H. (1988). Segregation of form, colour, movement and depth: Anatomy, physiology and perception. Science, 240, 740-749.

MaCLeOd, A., \& Summerfield, Q. (1987). Quantifying the contribution of vision to speech perception in noise. British Journal of Audiology, 12, 131-141.

Macleod, A., \& Summerfield, Q. (1990). A procedure for measuring auditory and audio-visual speech-reception thresholds for sentences in noise: Rationale, evaluation, and recommendations for use. British Journal of Audiology, 24, 29-43.

MARr, D., \& Nishihara, H. K. (1978). Representation and recognition of the spatial organization of three-dimensional shapes. Proceedings of the Royal Society of London: Series B, 200, 269-294.

Massaro, D. W., \& Cohen, M. M. (1990). Perception of synthesized audible and visible speech. Psychological Science, 1, 55-63.

Massaro, D. W., \& Cohen, M. M. (1996). Perceiving speech from inverted faces. Perception \& Psychophysics, 58, 1047-1065.

MCGurk, H., \& MACDonald, J. (1976). Hearing lips and seeing voices. Nature, 264, 746-748.

Metha, A. B., Vingrys, A. J., \& Badcock, D. R. (1994). Detection and discrimination of moving stimuli: The effects of color, luminance, and eccentricity. Journal of the Optical Society of America A, 11, 16971709.

MidDleweerd, M. J., \& Plomp, R. (1987). The effect of speechreading on the speech-reception threshold of sentences in noise. Journal of the Acoustical Society of America, 82, 2145-2147.

Montgomery, A. A., \& JACKSON, P. L. (1983). Physical characteristics of the lips underlying vowel lipreading performance. Journal of the Acoustical Society of America, 73, 2134-2144.

Muller, E. M., \& MacLeod, G. (1982, April). Perioral biomechanics and its relation to labial motor control. Paper presented at the meeting of the Acoustical Society of America, Chicago.

Munhall, K. G., \& Vatikiotis-Bateson, E. (1998). The moving face during speech communication. In R. Campbell, B. Dodd, \& D. Burnham (Eds.), Hearing by eye: Part 2. The psychology of speechreading and audiovisual speech (pp. 123-139). London: Taylor \& Francis.

OHala, J. J. (1975). The temporal regulation of speech. In G. Fant \& M. A. A. Tatham (Eds.), Auditory analysis and perception of speech (pp. 431-453). London: Academic Press.

Ostergaard, A. L., \& Davidoff, J. B. (1985). Some effects of color on naming and recognition of objects. Journal of Experimental Psychology: Learning, Memory, \& Cognition, 11, 579, 579-587.

Papathomas, T. V., Gorea, A., \& Julesz, B. (1991). Two carriers for motion perception: Color and luminance. Vision Research, 31, 1883-1991.

PETAJAN, E. D. (1984). Automatic lip reading to enhance speech recognition. In Proceedings of the IEEE Communications Society (pp. 265272). Washington, DC: IEEE.

Price, C. J., \& Humphreys, G. W. (1989). The effects of surface detail on object categorization and naming. Quarterly Journal of Experimental Psychology, 41A, 797-828.

Ramachandran, V. S. (1988). Perception of shape from shading. $\mathrm{Na}$ ture, 331, 163-166.

Ramachandran, V. S., \& Gregory, R. L. (1978). Does colour provide an input to human motion perception? Nature, 275, 55-56.

Reisberg, D., McLean, J., \& Goldfield, A. (1987). Easy to hear but hard to understand: A lipreading advantage with intact auditory stimuli. In B. Dodd \& R. Campbell (Eds.), Hearing by eye: The psychology of lip reading (pp. 97-113). London: Erlbaum. 
RoNNEBERG, J. (1993). Cognitive characteristics of skilled tactiling: The case of G.S. European Journal of Cognitive Psychology, 5, 19-33.

Rosenblum, L. D., Johnson, J., \& Saldaña, H. M. (1996). Visual kinematic information for embellishing speech in noise. Journal of Speech \& Hearing Research, 39, 1159-1170.

Rosenblum, L. D., \& Saldaña, H. M. (1992). Discrimination tests of visually influenced syllables. Perception \& Psychophysics, 52, 461-473.

Rosenblum, L. D., \& SAldañA, H. M. (1996). An audiovisual test of kinematic primitives for visual speech perception. Journal of Experimental Psychology: Human Perception \& Performance, 22, 318-331.

Rosenblum, L. D., \& Saldaña, H. M. (1998). Time-varying information for visual speech perception. In R. Campbell, B. Dodd, \& D. Burnham (Eds.), Hearing by eye: Part 2. The psychology of speechreading and audiovisual speech (pp. 61-81). London: Taylor \& Francis.

Samuelsson, S., \& RonNeberG, J. (1993). Implicit and explicit use of scripted constraints in lip reading. European Journal of Cognitive Psychology, 5, 201-233.

Sekuler, R., \& Blake, R. (1994). Perception. New York: McGraw-Hill.

Smeele, P. M. T., Hahnlen, L. D., Stevens, E. B., Kuhl, P K., \& Meltzoff, A. N. (1995). Investigating the role of specific facial information in audiovisual speech perception [Abstract]. Journal of the Acoustical Society of America, 98(5, Pt. 2), 2983.

STONE, L. (1957). Facial clues of context in lip reading. In John Tracy Clinic, Los Angeles Research Papers (Vol. 5, pp. 36-45). Los Angeles: John Tracy Clinic.

Sudman, J. A., \& Berger, K. W. (197l). Two-dimension vs. three dimension viewing in speech reading. Journal of Communication Disorders, 4, 195-198.

Sumby, W. H., \& Pollack, I. (1954). Visual contribution to speech intelligibility in noise. Journal of the Acoustical Society of America, 26, 212-215.

Summerfield, A. Q. (1987). Some preliminaries to a comprehensive account of audiovisual speech perception. In B. Dodd \& R. Campbell (Eds.), Hearing by eye: The psychology of lip reading (pp. 3-51). London: Erlbaum.

Summerfield, A. Q. (1992). Lip reading and audiovisual speech perception. In V. Bruce, A. Cowey, A. W. Ellis, \& D. I. Perrett (Eds.), Processing the facial image (pp. 71-78). Oxford: Oxford University Press.
Summerfield, Q., Macleod, A., McGrath, M., \& Brooke, M. (1989). Lips, teeth, and the benefits of lipreading. In A. W. Young \& H. D. Ellis (Eds.), Handbook of research on face processing (pp. 223233). Amsterdam: Elsevier.

Summerfield, Q., \& McGrath, M. (1984). Detection and resolution of audiovisual incompatibility in the perception of vowels. Quarterly Journal of Experimental Psychology, 36A, 51-74.

TrosCianKo, T. (1994). Contribution of colour to the motion aftereffect and motion perception. Perception, 23, 1221-1231.

Vatikiotis-Bateson, E., Eigsti, l.-M., Yano, S., \& Munhall, K. G. (1998). Eye movement of perceivers during audiovisual speech perception. Perception \& Psychophysics, 60, 926-940.

Walden, B. E., Prosek, R. A., Montgomery, A. A., Scherr, C. K., \& JONES, C. J. (1977). Effects of training on the visual recognition of consonants. Journal of Speech \& Hearing Research, 20, 130-145.

Walden, B. E., Prosek, R. A., \& Worthington, O. W. (1974). Predicting audiovisual consonant recognition performance of hearingimpaired adults. Journal of Speech \& Hearing Research, 17, 270-278.

Wurm, L. H., Legge, G. E., Isenberg, L. M., \& LuebKer, A. (1993). Color improves object recognition in normal and low vision. Journal of Experimental Psychology: Human Perception \& Performance, 19, 899-911.

\section{NOTES}

1. Presenting auditory speech stimuli with no face at all would have made it impossible to examine the extent to which visual speech affected congruent and incongruent auditory speech recognition above and beyond any effects of presenting merely a facial stimulus. Consequently, stimuli in the auditory speech condition were presented with a static face to provide an appropriate baseline.

2. Errors in the auditory-only condition arose mainly from confusion of the syllables / ba/ and /va/. These two syllables have been shown previously to be fragile with respect to correct identification when presented in the auditory modality alone (e.g., Massaro \& Cohen, 1996).

(Manuscript received January 20, 1999; revision accepted for publication November $4,1999$. 